Discrete Comput Geom 27:485-499 (2002)

DOI: $10.1007 / \mathrm{s} 00454-001-0093-0$

\title{
The Mean Number of Extreme Lines in a Convex Hull of Lines
}

\author{
John Gates \\ School of Computing and Mathematical Sciences, University of Greenwich, \\ 30 Park Row, Greenwich, London SE10 9LS, England \\ jgates@gre.ac.uk
}

\begin{abstract}
The dual problem of the mean number of extreme lines among $n$ chosen at random from a convex polygon of lines is defined, evaluations made for $n=4,6,20$ and the asymptotic mean are shown to be the same as in the classic case of $n$ points.
\end{abstract}

\section{Introduction}

The aim of this paper is to formulate the problem and approaches to finding the mean number of extreme lines amongst $n$ chosen at random from within a convex polygon of lines. It is the dual of the classical problem of the mean number of vertices of the convex hull of $n$ points. These mean values are obtained explicitly (with some numerical integration) for $n=4,6$ and 20 and found to be very close to the corresponding classical results. The asymptotic mean agrees with the same basic result found by Rényi and Sulanke (1963). The framework for convexity and convex hulls of line sets has been previously defined in Gates (1993), but a minimal description is given here.

The classical problem involves $n$ points chosen uniformly randomly and independently inside a convex body, $K$; let $e_{n}^{\mathrm{c}}(K)$ denote the expected number of vertices of the convex hull and let $A_{n}(K)$ denote its expected area. Discussion of $e_{n}^{\mathrm{c}}(K)$ in $d=2$ and then in higher dimensions is one of the oldest problems of geometric probability. For the planar case, finding $e_{4}^{\mathrm{c}}(K)$ is equivalent to Sylvester's problem of finding the probability that the convex hull of four points is a quadrilateral; this is also equivalent to finding $A_{3}(K)$. Results on the computation of $e_{4}(K)$ for various simple $K$ (such as triangle, quadrilateral, regular polygon, ellipse) can be found in Santaló (1976) and Buchta (1985). If $K$ is a quadrilateral of unit area with diagonals dividing $K$ into triangles of areas $\Delta_{1}, 1-\Delta_{1}$ and $\Delta_{2}, 1-\Delta_{2}$, then an old result of Herglotz is that $A_{3}(K)=\frac{1}{12}-\frac{1}{9} \Delta_{1}\left(1-\Delta_{1}\right) \Delta_{2}\left(1-\Delta_{2}\right)$. Recently Buchta and Reitzner (1997b) found an expression for $A_{n}(K)$ when $K$ is a planar convex polygon in terms of the areas of 
polygons associated with $K$. In 1923 Blaschke showed that $e_{4}^{\mathrm{c}}(K)$ is least for the case that $K$ is a triangle. Groemer (1974) proved $e_{d+1}^{\mathrm{c}}(K)$ is greatest for $K$ an ellipsoid (only) in $d$ dimensions. Recently Giannopoulos (1992) showed that $A_{n}(K)<A_{n}$ (triangle) unless $K$ is a triangle (this maximum value is given in a later section here). In the threedimensional case the expected volume of four uniform points in a simplex has been computed by Mannion (1994) and by Butcha and Reitzner (1992); it is often surmised, but not proven, that this expected volume is greater than for any other convex body.

In connection with this paper, the main reference is Rényi and Sulanke (1963); in particular they obtained an expression for the asymptotic behaviour of $e_{n}^{\mathrm{c}}(K)$, for $K$ a planar polygon (also in the case of $K$ with smooth boundary). In arbitrary dimension, the asymptotics of $e_{n}^{\mathrm{c}}(K)$ (as $n \rightarrow \infty$ ) was explored by Dwyer (1988), Affentranger and Wieacker (1991) and then Bárány and Buchta (1993), for polytopes, and also by Bárány and Larman (1988) for arbitrary convex bodies. A quite different, more stochastic, approach was made by Groeneboom (1988) for planar polygons; by showing that the convex hull vertices, after rescaling, become essentially a Poisson point process near any of the parent vertices, he showed that the number of hull vertices is asymptotically Normal, obtaining the asymptotic variance as well as the mean. A similar approach in Cabo and Groeneboom (1994) obtained results for the boundary length and area of the convex hull. Summaries of these results and more references can be found in survey papers by Schneider (1988), by Weil and Wieacker (1993) and also by Schneider (1997); the latter contains outline references and also some open problems. A brief recent survey of extremal results can be found in Bauer and Schneider (1995). Buchta and Reitzner (1997a, b) use their exact expression for $A_{n}(K)$, for $K$ a planar polygon, to obtain an asymptotic expansion for $e_{n}^{\mathrm{c}}(K)$, up to a term $n^{-2}$.

\section{Formulation and Some Finite Results}

We work in the set $\Gamma$ of directed lines in $R^{2}$. Each $g \in \Gamma$ has a left-hand and right-hand closed half-space, $L(g)$ and $R(g)$, respectively. We deal with the lines selected uniformly (with respect to the invariant measure) at random from the compact convex hull, $H_{0}$, of $r$ lines $g_{1}, g_{2}, \ldots, g_{r}$. If $L_{0}$ is the intersection of all the $L\left(g_{i}\right)$ and $R_{0}$ is the intersection of all the $R\left(g_{i}\right)$, then $H_{0}$ consists of all lines $g$ such that $L_{0} \subset L(g)$ and $R_{0} \subset R(g)$ : let $H_{0}$ have measure $\mu_{0} . L_{0}$ and $R_{0}$ are two infinite polygonal sets with edges formed by the $g_{i}$. If no two elements of $g_{1}, g_{2}, \ldots, g_{r}$ are parallel, to check that $g \in H_{0}$ it suffices to check that the vertices of $L_{0}$ are within $L(g)$ and the vertices of $R_{0}$ are within $R(g)$.

If $n \geq 3$ independent uniform random lines $G_{1}, G_{2}, \ldots, G_{n}$ are chosen from $H_{0}$ they have a convex hull $H\left(\subset H_{0}\right)$ : let $L$ and $R$ denote the left-hand and right-hand sets of $H$ and let $N_{n}$ be the number of extreme lines amongst these $n$ (where $G_{i}$ is extreme if it is outside the convex hull of the other lines). $L$ is the intersection of half-planes; suppose it has $l$ vertices; suppose that $R$ has $r$ vertices. The extreme lines of $H$ are the lines joining consecutive vertices of $L(l-1$ of these) and the lines joining consecutive vertices of $R$ ( $r-1$ of these) together with the two support lines from $L$ to $R$ (which form edges of $L$ and $R$ ). The number of these extreme lines is $N_{n}=l+r$, the number of vertices of $L \cup R$, each point of which is of the form $G_{i} \cap G_{j}$. In the classic case of $n$ points in a convex region a useful fact used by Rényi and Sulanke (and many others) is that two 
points form an edge of the convex hull if and only if the other $n-2$ points all fall on one side or all on the other side of the line joining them. The dual of this fact is that $G_{i} \cap G_{j}$ will form a vertex of $L \cup R$ if the other $n-2$ lines are all positive (anticlockwise) or all negative (clockwise) about this point.

Let $P=G_{1} \cap G_{2}$ and $p_{+}(P)$ denote the probability that a random line of $H_{0}$ is positive about $P$ and $p_{-}(P)=1-p_{+}(P)$. Then the probability that $P$ forms a vertex of $L \cup R$ is $\left(p_{+}(P)\right)^{m}+\left(p_{-}(P)\right)^{m}$, where $m$ is used throughout to denote $n-2$ (so $m \geq 1)$. We calculate $p_{+}(P)$ from the measure of lines with $L_{0} \cup\{P\} \subset L(g)$ and with $R_{0} \subset R(g)$; this measure can be expressed in terms of interpoint distances. As $N_{n}=\sum_{1<i<j \leq n} I(i, j)$, where $I(i, j)=1$ if $G_{i} \cap G_{j}$ is a vertex $L \cup R$ and 0 otherwise, the expected number of extreme lines is

$$
\begin{aligned}
e_{n} & =E\left(N_{n}\right)=\left(\begin{array}{l}
n \\
2
\end{array}\right) E(I(1,2)) \\
& =\left(\begin{array}{l}
n \\
2
\end{array}\right) \int_{R^{2}-L_{0} \cup R_{0}}\left(\left(p_{+}(P)\right)^{m}+\left(p_{-}(P)\right)^{m}\right) f(P) d v,
\end{aligned}
$$

where $d v$ denotes the Lebesgue measure in the plane and $f(P)$ is the probability density function of the intersection point of two random lines from $H_{0}$. In Gates (1993) the density, for $P$ in $R^{2}-\left(L_{0} \cup R_{0}\right)$, was shown to be

$$
f(P)=2 \mu_{0}^{-2}(\omega-\sin \omega),
$$

where $\omega$ is the angular range of the pencil of lines of $H_{0}$ through $P$.

Some elementary algebraic identities are relevant here. If we define polynomials

$$
u_{m}(x)=x^{m}+(1-x)^{m},
$$

then, for odd $m, u_{m}$ is a linear combination of $u_{r}$ for $r<m$. For example,

$$
\begin{gathered}
u_{3}=1 / 2\left(3 u_{2}-1\right) ; \quad u_{5}=1 / 2\left(5 u_{4}-5 u_{2}+2\right) ; \\
u_{7}=1 / 4\left(14 u_{6}-35 u_{4}+42 u_{2}-17\right) ;
\end{gathered}
$$

which imply

$$
\begin{gathered}
e_{5}=5 / 2\left(e_{4}-2\right), \quad e_{7}=7 / 4\left(2 e_{6}-5 e_{4}+12\right), \\
e_{9}=3 / 2\left(3 e_{8}-14 e_{6}+42 e_{4}-102\right) .
\end{gathered}
$$

Each point $P$ of $R^{2}-\left(L_{0} \cup R_{0}\right)$ has two support points on the boundary of $L_{0} \cup R_{0}$, the two vertices at the extremes of the pencil of lines through $P$. Thus $R^{2}-\left(L_{0} \cup R_{0}\right)$ is partitioned into compartments, each corresponding to a pair of vertices. Some of these compartments are split into two components. Points within a compartment will be parametrized by a pair of angles from the corresponding vertex pair.

Consider the case when the parent polygon is a triangle $(r=3)$. Let $g_{1}, g_{2}, g_{3}$ be the three generating lines (no two parallel) labelled at increasing orientations and assume that $g_{2}$ is positive about $g_{1} \cap g_{3}$. To generate a triangle the three lines have 


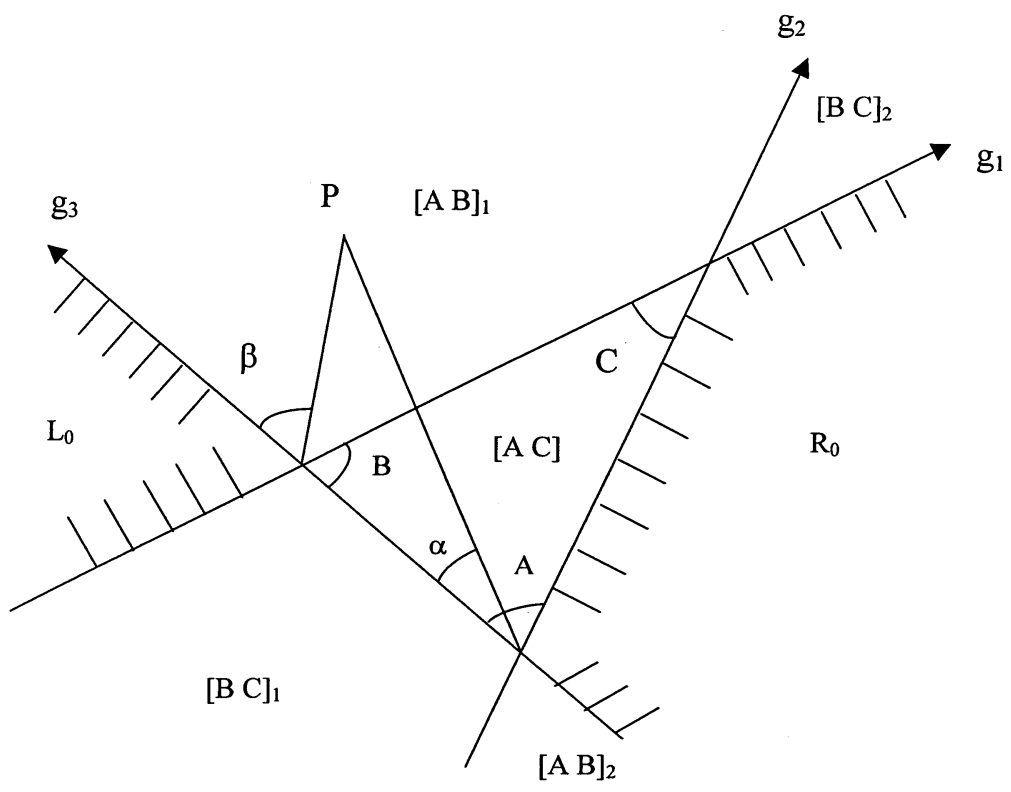

Fig. 1

to be non-concurrent and with orientations strictly within a semicircle. Let $A, B, C$ denote both the points of intersection $g_{2} \cap g_{3}, g_{1} \cap g_{3}, g_{1} \cap g_{2}$, respectively, and also the internal angles of the triangle formed (see Fig. 1). Figure 1 shows the three compartments $[A B],[A C]$ and $[B C]$, with $[A B]$ split into $[A B]_{1}$ and $[A B]_{2}$ and $[B C]$ into $[B C]_{1}$ and $[B C]_{2}$. To avoid cumbersome notation we use an adaptive system. For $P \in[A B]$ let $\alpha$ be the angle from $g_{3}$ back to $A P$ and let $\beta$ be the angle from $g_{3}$ back to $B P$; with $0 \leq \beta \leq \pi-B$ and $P \in[A B]_{1}$ if $\alpha \leq \beta$. For $P \in[A C]$ let $\alpha$ be the angle from $g_{2}$ forward to $A P$ and let $\gamma$ be the angle from $g_{2}$ back to $P C$; with $0 \leq \alpha \leq A$ and $0 \leq \gamma \leq C$. For $P \in[B C]$ let $\beta$ be the angle from $g_{1}$ forward to $P B$ and let $\gamma$ be the angle from $g_{1}$ forward to $P C$, with $0 \leq \beta \leq \pi-B$ and $0 \leq \gamma \leq C$ and $p \in[B C]_{1}$ if $\gamma \leq \beta$.

For $P \in[A B]$ let $p_{*}$ be the probability that a random line has the same orientation about $P$ as $g_{3}$, so that $p_{*}$ is $p_{-}(P)$ on $[A B]_{1}$ and $p_{*}=p_{+}(P)$ on $[A B]_{2}$. On $[A C]$ and $[B C] p_{*}$ is defined as $p_{+}(P)$ or $p_{-}(P)$ by the orientation of $g_{2}$ and $g_{1}$, respectively.

Suppose the triangle has been sized to give the total line measure

$\mu_{0}=|A B|+|B C|-|A C|=1, \quad$ so that $\quad|A B|=\sin (C)(\sin (A)+\sin (C)-\sin (B))^{-1}$.

For $P \in[A B], p_{*}=|A B|+|B P|-|A P|=|A B| U_{A B}$, where $U_{A B}=1-(\sin (\beta)-$ $\sin (\alpha)) / \sin (\beta-\alpha)$. Then the probability of $m$ lines passing all on one side of $P$ is

$$
\left.p_{*}^{m}+\left(1-p_{*}\right)^{m}=\left(|A B| U_{A B}\right)\right)^{m}+\left(1-|A B| U_{A B}\right)^{m} .
$$


If the Cartesian co-ordinates of $A$ and $B$ are $\left(a_{1}, a_{2}\right)$ and $\left(b_{2}, b_{2}\right)$, then those of $P$ are

$$
\begin{array}{r}
(x, y)=\left(a_{1}, a_{2}\right)+\frac{\sin \beta}{\sin (\beta-\alpha)}\left(\left(b_{1}-a_{1}\right) \cos \alpha+\left(b_{2}-a_{2}\right) \sin \alpha,\right. \\
\left.\left(b_{2}-a_{2}\right) \cos \alpha-\left(b_{1}-a_{1}\right) \sin \alpha\right)
\end{array}
$$

for $0 \leq \alpha \leq A, 0 \leq \beta \leq \pi-B$.

We can transform the probability element, from (2), to $\alpha-\beta$ co-ordinates to obtain $p_{A B}(\alpha, \beta) d \alpha d \beta$, where

$$
p_{A B}(\alpha, \beta)=2|A B|^{2} q(\beta-\alpha) \sin (\alpha) \sin (\beta),
$$

with the function $q$ defined on $[0, \pi)$ by

$$
q(x)= \begin{cases}(x-\sin (x)) / \sin ^{3}(x), & 0<x<\pi, \\ \frac{1}{6}, & x=0 .\end{cases}
$$

For $P \in[B C]$ the same expressions apply, with $A B$ changed to $B C$ and $\alpha$ replaced by $\gamma$ to form $U_{B C}$ and $p_{B C}(\beta, \gamma)$.

For $P \in[A C]$ we have $p_{*}=|A C| U_{A C}$, where $U_{A C}=(\sin (\alpha)+\sin (\gamma)) / \sin (\alpha+\gamma)-1$ and the corresponding probability density is

$$
p_{A C}(\alpha, \gamma)=2|A C|^{2} q(\alpha+\gamma) \sin (\alpha) \sin (\gamma) .
$$

Determining $e_{n}$ requires the evaluation of

$$
\begin{aligned}
\iint\left(\left(|A B| U_{A B}\right)^{m}+\left(1-|A B| U_{A B}\right)^{m}\right) p_{A B}(\alpha, \beta) d \alpha d \beta \\
+\iint\left(\left(|B C| U_{B C}\right)^{m}+\left(1-|B C| U_{B C}\right)^{m}\right) p_{B C}(\beta, \gamma) d \beta d \gamma \\
+\iint\left(\left(|A C| U_{A C}\right)^{m}+\left(1-|A C| U_{A C}\right)^{m}\right) p_{A C}(\alpha, \gamma) d \alpha d \gamma
\end{aligned}
$$

It is possible to perform one stage of integration analytically by making the substitutions $\omega=\beta-\alpha$ and $t=\beta+\alpha$, but the second stage has to be carried out numerically. Evaluations of (8) were carried out for two triangles; Triangle 1 had $A=B=C=\pi / 3$ and Triangle 2 had $A=C=\pi / 4$ and $B=\pi / 2$; values of $n=m+2=4,6$ and 20 were used and the results shown in Table 1. Corresponding values for $n=5$ and 7 can be deduced using (3).

In the dual case there is also a special case that does not occur classically, this is when two of the parent lines generating $H_{0}$ are parallel. Suppose that $g_{1}$ and $g_{2}$ are parallel, distance $h$ apart, with $g_{2} \subset L\left(g_{1}\right)$, and that $g_{3}$ makes an angle $A$ with them and crosses from $R\left(g_{1}\right)$ to $L\left(g_{1}\right)$. Denote $g_{2} \cap g_{3}$ by $A$ and $g_{1} \cap g_{3}$ by $B$ and regard the point at infinity $g_{1} \cap g_{2}$ as " $C$ ". Again we can decompose $R^{2}-\left(L_{0} \cup R_{0}\right)$ into three compartments with $[A B]$ having two components. The measure of lines in this "vertical" triangle can be easily shown to be $h \tan (A / 2)$, so that $h=\cot (A / 2)$ if we assumed this measure has been sized to 1 . For $P \in[A B]$ the expressions for $p_{*}$ and the density for $P$ are as before. 
Table 1

\begin{tabular}{lccc}
\hline \multicolumn{1}{c}{ Parent polygon } & $n=4$ & $n=6$ & $n=20$ \\
\hline Triangle 1 & 3.66706 & 4.56833 & 7.10502 \\
Triangle 2 & 3.66745 & 4.56995 & 7.11226 \\
Vertical triangle & 3.66744 & 4.56991 & 7.11267 \\
Classical triangle & 3.66667 & 4.56667 & 7.09548 \\
\hline
\end{tabular}

For $P \in[A C]=L\left(g_{1}\right) \cap R\left(g_{2}\right) \cap L\left(g_{3}\right)$ we parametrize by the angle $\alpha$ of $A P$ with $g_{2}$ and the distance $y$ of $P$ from $g_{2}$. For such a $P, p_{*}=p_{-}(P)$ is calculated as $y \tan (y / 2)$ and the probability element is

$$
d P=2(\alpha-\sin (\alpha)) y(\sin (\alpha))^{-2} d \alpha d y
$$

for $0 \leq \alpha \leq A$ and $0 \leq y \leq h$. The integration of $p_{*}^{m}+\left(1-p_{*}\right)^{m}$ is then

$$
\int_{0}^{A} 2(\alpha-\sin (\alpha))(\sin (\alpha))^{-2} \int_{0}^{h} y\left[(y \tan (\alpha / 2))^{m}+(1-y \tan (\alpha / 2))^{m}\right] d y d \alpha .
$$

The integral for the $B C$ case is the same. Numerical evaluations were made for $A=\pi / 2$ (and so $h=1$ ) for $n=4,6$ and 20 and these are included in Table 1 .

For comparison with the classical case we give the values of $e_{n}^{\mathrm{c}}$ for $n$ points in a (any) triangle. From the original Rényi and Sulanke (1963) paper, where we can assume an equilateral triangle (and put back the neglected term), we easily find

$$
\begin{aligned}
e_{n}^{\mathrm{c}}(\text { triangle }) & =4\left(\begin{array}{l}
n \\
2
\end{array}\right) \int_{0}^{1} \int_{0}^{1}\left[(1-x y)^{m}+(x y)^{m}\right] x y d x d y \\
& =2 \sum_{k=1}^{n-1} \frac{1}{k},
\end{aligned}
$$

which can also be found in Buchta (1985).

\section{Asymptotic Behaviour of $e_{n}$ for a Triangle}

In the classic case of $n$ points at random in a convex polygon with $r$ vertices Rényi and Sulanke (1963) established that $e_{n}^{\mathrm{c}} \sim \frac{2}{3} r \ln (n)$, as $n \rightarrow \infty$. We denote by $\varphi(m)$ the function $\ln (m) / m^{2}$; terms which are $o(\varphi(m))$ will be described as negligible. We consider

$$
\iint\left(p_{*}^{m}+\left(1-p_{*}\right)^{m}\right) f(x, y) d x d y
$$

for large $m$. The method of analysis is the classical Laplace method (see Wong, 1989) which says that (12) will be dominated by the region where $p_{*}$ is close to 1 or 0 , this is where $P$ is near the boundary of $R^{2}-\left(L_{0} \cup R_{0}\right)$. We make approximations to (12) which then behave like (11). The details of the error bounds of the approximation are lengthy and are shown in one case and then omitted. The following lemmas allow bounds for this approximation to be established. 


\section{Lemma 1.}

(a) With $q$ defined in (6) the function

$$
h(t)= \begin{cases}t^{-2}\left(q(t)-\frac{1}{6}\right), & t>0, \\ \frac{3}{40}, & t=0\end{cases}
$$

is continuous and increasing on $[0, \pi)$ and $h(1)<\frac{1}{10}$.

(b) $\left(1-m x^{2}\right) e^{-m x} \leq(1-x)^{m} \leq e^{-m x}$ for $0 \leq x \leq 1, m \geq 1$.

Proof. For (a), for $t$ near 0 ,

$$
\begin{aligned}
h(t) & \left.=\left(t^{3} / 6-t^{5} / 120 \cdots\right)-\left(t^{3}-t^{5} / 120 \cdots\right) / 6\right) t^{-2}\left(t-t^{3} \cdots\right)^{-3} \\
& =\frac{3}{40}+O(t),
\end{aligned}
$$

and the continuity at $t=0$ is clear. To prove that $h$ is increasing we show that the derivative is positive on $(0, \pi)$ :

$$
\begin{aligned}
r(t)= & h^{\prime}(t)\left(t^{3} \sin ^{4}(t)\right) \\
= & \frac{9}{8}+t \sin (2 t)-t \sin (t)-3 t^{2} \cos (t)-\frac{7}{6} \cos (2 t)+\cos (4 t) / 24 \\
\geq & \frac{9}{8}+t\left(2 t-4 / 3 t^{3}+4 / 15 t^{5}-8 / 315 t^{7}\right) \\
& -t\left(t-t^{3} / 6+t^{5} / 120-t^{7} / 5040+t^{9} / 9 !\right) \\
& -3 t^{2}\left(1-t^{2} / 2+t^{4} / 24-t^{6} / 6 !-t^{8} / 8 !\right) \\
& -\frac{7}{6}\left(1-2 t^{2}+2 t^{4} / 3-4 t^{6} / 45+2 t^{8} / 315\right) \\
& +\left(1-8 t^{2}+32 / 3 t^{4}-256 / 45 t^{6}+512 t^{8} / 315-4096 t^{10} / 14175\right) \\
= & 33 t^{8} / 840-16489 t^{10} / 1360800 .
\end{aligned}
$$

This lower bound is positive on $(0, \pi / 2)$ and so $h^{\prime}(t)$ is positive on this interval. For a bound on $[\pi / 2, \pi)$ write $t$ as $\pi / 2+y$, then

$$
\begin{aligned}
r(t)= & \frac{9}{8}-(y+\pi / 2) \sin (2 y)-(y+\pi / 2) \cos (y)+3(y+\pi / 2)^{3} \sin (y) \\
& +\frac{7}{6} \cos (2 y)+\cos (4 y) / 24 \\
\geq & \frac{9}{8}-(y+\pi / 2)(2 y)-(y+\pi / 2)\left(1-y^{2} / 2+y^{4} / 24\right)+3(y+\pi / 2)^{2}\left(y-y^{3} / 6\right) \\
& +\frac{7}{6}\left(1-2 y^{2}\right)+\left(1-8 y^{2}\right) / 24 \\
= & \left(\frac{7}{3}-\pi / 2\right)+y\left(3 \pi^{2} / 4-\pi-1\right)+y^{2}\left(13 \pi / 4-\frac{14}{3}\right)+y^{3}\left(\frac{7}{2}-\pi^{2} / 8\right) \\
& -25 \pi y^{4} / 48-13 y^{5} / 24 \\
> & 0.5+3 y+5 y^{2}+2 y^{3}-2 y^{4}-y^{5} \\
\geq & 0.5+\left(12 / \pi^{2}+10 / \pi+2\right) y^{3}-2 y^{4}-y^{5} \\
> & 0.5+y^{3}\left(7-(y+1)^{2}\right) \geq 0.5,
\end{aligned}
$$

for $y<\pi / 2$ and the increasing nature of $h(t)$ is established. 
For (b) we have, as standard,

$$
(1-x)^{m} \leq e^{-m x} \leq(1+x)^{-m},
$$

which gives the right-hand side of (b). Also $\left(1-m x^{2}\right) \leq\left(1-x^{2}\right)^{m}$ so

$$
\left(1-m x^{2}\right) e^{-m x} \leq\left(1-x^{2}\right)^{m}(1+x)^{-m}=(1-x)^{m} .
$$

A Corollary to Lemma 1 is that (from (5)) $p_{A B}(\alpha, \beta) \leq K \alpha \beta$ and (from (7)) $p_{A C}(\alpha, \gamma)$ $\leq K \alpha \gamma$, where $K$ is some constant that depends on $A, B, C$.

Lemma 2. If

$$
U(x, y)= \begin{cases}1-(\sin (x)-\sin (y)) / \sin (x-y) & \text { for } x \neq y, \\ 1-\cos (x) & \text { for } x=y,\end{cases}
$$

then

(a) $\left(2 / \pi^{2}\right) x y \leq U(x, y)$ for $0 \leq x<\pi, 0 \leq y<\pi$;

(b) $x y\left(\frac{1}{2}-x^{2} / 24\right) \leq U(x, y) \leq x y\left(\frac{1}{2}+x^{2} / 2\right)$ for $0 \leq y \leq x \leq 1$.

Proof. Let $R(x, y)=U(x, y) / x y$, as this is symmetrical in $x$ and $y$ consider only $0 \leq y \leq x$. Using basic trigonometric identities this can be written

$$
R(x, y)=\frac{2 \sin (x / 2) \sin (y / 2)}{x y \cos ((x-y) / 2)} .
$$

As a function of $y$ on $[0, x]$, with $x$ fixed, this is decreasing (as $y^{-1} \sin (y / 2)$ and $(\cos ((x-$ $y) / 2))^{-1}$ are decreasing). Thus

$$
2(\sin (x / 2) / x)^{2} \leq R(x, y) \leq \tan (x / 2) / x .
$$

The lower bound is a decreasing function of $x$ on $[0, \pi]$, hence showing that $R(x, y) \geq$ $2 / \pi^{2}$, establishing (a).

For (b) we note that $2(\sin (x / 2) / x)^{2} \geq \frac{1}{2}-x^{2} / 24$ for all $x$ in $[0, \pi]$, giving the lower bound. Also

$\tan (x / 2) / x=\sin (x / 2) /(x \cos (x / 2)) \leq 1 /(2 \cos (x / 2)) \leq 1 /\left(2\left(1-x^{2} / 8\right)\right) \leq\left(1+x^{2}\right) / 2$,

with the last inequality valid for $x^{2} \leq 7$. Hence the upper bound of (b) will be valid for $0 \leq y \leq x \leq 1$.

Consider the general triangle and $P \in[A B]$. The function $p_{*}=|A B| U(\alpha, \beta)$ is continuous on $[0, A] \times[0, \pi-B]$, is always non-negative on this domain, attains its maximum of 1 only at $\alpha=A$ and $\beta=\pi-B$ and has partial derivatives

$$
\begin{aligned}
& D_{1} p_{*}(\alpha, \beta)=|A B|(1-\sin \beta \cos (\alpha-\beta))\left(\sin ^{2}(\alpha-\beta)\right)^{-1}, \\
& D_{2} p_{*}(\alpha, \beta)=|A B|(1-\sin \alpha \cos (\alpha-\beta))\left(\sin ^{2}(\alpha-\beta)\right)^{-1} .
\end{aligned}
$$


On the sub-domain $A-C / 2 \leq \alpha \leq A, \pi-B-C / 2 \leq \beta \leq \pi-B$ we have $C / 2 \leq \beta-\alpha$ and so both partial derivatives are at least $|A B|\left(2 \cos ^{2}(C / 4)\right)^{-1}$. Using the Mean Value Theorem it follows that, on this sub-domain, $1-p_{*} \geq|A B|\left(2 \cos ^{2}(C / 4)\right)^{-1}(A-\alpha+\pi-$ $B-\beta)$. Away from this corner, on $[0, A] \times[0, \pi-B-C / 2] \cup[0, A-C / 2] \times[0, \pi-B]$, the function $(A-\alpha+\pi-B-\beta)\left(1-p_{*}\right)^{-1}$ is positive and continuous and so bounded above by $1 / m_{2}$.

Hence if $k_{1}$ is the smaller of $|A B|\left(2 \cos ^{2}(C / 4)\right)^{-1}$ and $m_{2}$ we have, on all of $[0, A] \times$ $[0, \pi-B]$, that

$$
p_{*} \leq 1-k_{1}(A-\alpha+\pi-B-\beta)
$$

with the constant $k_{1}>0$. Consequently, integrating over this domain,

$$
\begin{aligned}
\iint p_{*}^{m} p_{A B}(\alpha, \beta) d \alpha d \beta & \leq \iint \exp \left(-m k_{1}(A-\alpha+\pi-B-\beta)\right) K \pi^{2} d \alpha d \beta \\
& \leq K \pi^{2} /\left(m^{2} k_{1}^{2}\right)=o(\varphi(m)) .
\end{aligned}
$$

Thus the integral of $p_{*}^{m}$ makes a negligible contribution to (8) for large $m$.

To examine the integral of $\left(1-p_{*}\right)^{m}$ we will find that the dominant part comes where $\alpha$ and $\beta$ are small. Let $c(m)$ denote $(\log (m))^{-1}$ and suppose that $m$ is large enough to ensure that $0<c(m)<\min (1, A, \pi-B)$. On $D_{1}=[c(m), A] \times[0, \pi-B]$, from Lemma 1,

$$
\iint\left(1-p_{*}\right)^{m} p_{A B}(\alpha, \beta) d \alpha d \beta \leq \iint e^{-m p_{*}} K \alpha \beta d \alpha d \beta \leq \iint K e^{-m k_{2} \alpha \beta} \alpha \beta d \alpha d \beta,
$$

where $k_{2}|A B| / \pi^{2}$. Using the substitutions $p=\alpha \beta$ and $u=\beta / \alpha$ the last integral becomes

$$
\begin{aligned}
& \int_{0}^{A(\pi-B)} K e^{-m p k_{2}} p\left\{\int_{p / A}^{\min \left\{p / c(m)^{2},(\pi-B)^{2} / p\right\}}(2 u)^{-1} d u\right\} d p \\
&<K \ln (A / c(m)) \int_{0}^{\infty} p e^{-m p k_{2}} d p=K \ln (A / c(m)) /\left(m k_{2}\right)^{2}
\end{aligned}
$$

which is $o(\varphi(m)$. As the initial integral is positive this also implies that the same integral over the domain $[c(m), A] \times[c(m), \pi-B]$ and, by reversing the variables, over $[0, c(m)] \times[c(m), \pi-B]$ are negligible. This leaves us to consider the integral over the domain $[0, c(m)] \times[0, c(m)]$; by symmetry this is twice the integral over the domain $D_{2}=\{(\alpha, \beta): 0 \leq \beta \leq \alpha \leq c(m)\}$. for $p$.

The following results can be established by making the substitution $y=m k(m) p$

Lemma 3. Suppose that $\{k(m)\}$ is a sequence with limit $k$ as $m \rightarrow \infty$ :

(a) If $I(m)=\int_{0}^{c(m)^{2}} e^{-m k(m) p} p \log \left(c(m)^{2} / p\right) d p$, then $I(m) / \varphi(m) \rightarrow 1 / k^{2}$.

(b) If $J(m)=\int_{0}^{c(m)^{2}} e^{-m k(m) p} p^{3} \log \left(c(m)^{2} / p\right) d p$, then $I(m) / \varphi(m) \rightarrow 0$, as $m \rightarrow \infty$. 
Now consider

$$
A(m)=\iint_{D_{2}}\left(1-p_{*}\right)^{m} p_{A B}(\alpha, \beta) d \alpha d \beta .
$$

From Lemma 1 , as $\alpha-\beta \leq c(m) \leq 1$, we have

$$
\begin{aligned}
A(m) & \leq 2|A B|^{2} \iint_{D_{2}}\left(\frac{1}{6}+c(m)^{2} / 10\right) e^{-m p_{*}} \alpha \beta d \alpha d \beta \\
& \leq 2|A B|^{2}\left(\frac{1}{6}+c(m)^{2} / 10\right) \iint_{D_{2}} e^{-m k(m) \alpha \beta} \alpha \beta d \alpha d \beta,
\end{aligned}
$$

with $k(m)=|A B|\left(\frac{1}{2}-c(m)^{2} / 24\right)$. Again making the substitutions $p=\alpha \beta$ and $u=\beta / \alpha$ and performing the integration with respect to $u$ we obtain

$$
A(m) \leq 2|A B|^{2}\left(\frac{1}{6}+c(m)^{2} / 10\right) \int_{0}^{c(m)^{2}} e^{-m k(m) p} p \ln \left(c(m)^{2} / p\right) / 2 d p,
$$

by Lemma 3 we conclude that $\lim \sup (A(m) / \varphi(m)) \leq \frac{2}{3}$.

Also by Lemmas 1 and 2 we have that

$$
A(m) \geq 2|A B|^{2} \iint_{D_{2}} \frac{1}{6}\left(1-m l(m)^{2} p^{2}\right) e^{-m l(m) p} \alpha \beta\left(1-c(m)^{2} / 3\right) d \alpha d \beta,
$$

where $1(m)=|A B|\left(1+c(m)^{2}\right) / 2$. The same substitution leads to

$$
A(m) \geq 2|A B|^{2}\left(\frac{1}{6}-c(m)^{2} / 18\right) \int_{0}^{c(m)^{2}}\left(p-m l(m)^{2} p^{3}\right) e^{-m l(m) p}\left(\log \left(c(m)^{2} / p\right) / 2\right) d p .
$$

By Lemma 3 we obtain $\lim \inf (A(m) / \varphi(m)) \geq \frac{2}{3}$. The two inequalities imply that $\lim (A(m) / \varphi(m))=\frac{2}{3}$ and hence, combining domains, that

$$
\lim \left[1 /(\varphi(m))\left(\iint\left(\left(1-p_{*}\right)^{m}+p_{*}^{m}\right) p_{A B}(\alpha, \beta) d \alpha d \beta\right)\right]=\frac{4}{3} .
$$

For the $\mathrm{BC}$ term the derivation is the same, also giving the limit of $\frac{4}{3}$.

For $P \in A C, p_{*}$ is the probability of a random line being positive about $P$ so

$$
p_{*}=|A C|(\sin (\alpha)+\sin (\gamma)-\sin (\alpha+\gamma)) / \sin (\alpha+\gamma),
$$

which is near 0 if $P$ is near $g_{2}$ (small $\alpha$ or small $\gamma$ ) and is near 1 only if $P$ is near $B$. As in the reasoning of (13) and (14) this implies that the contribution of the integral of $p_{*}^{m}$ is asymptotically negligible. For $P$ near $g_{2}$,

$$
\left(1-p_{*}\right)^{m} \cong(1-|A C| \alpha \gamma / 2)^{m} \cong e^{-k_{3} \alpha \gamma},
$$


where $k_{3}=|A C| / 2$. Hence,

$$
\begin{aligned}
\int_{0}^{C} \int_{0}^{A}\left(1-p_{*}\right)^{m} p_{A C}(\alpha, \gamma) d \alpha d \gamma & \cong|A C|^{2} / 3 \int_{0}^{A . C} e^{-k_{3} m p} p \int_{p / c(m)^{2}}^{A^{2} / p}(1 / 2 u) d u d p \\
& \cong 4 / 3 \varphi(m) .
\end{aligned}
$$

Consequently we see that each of the integrals of $p_{*}^{m}+\left(1-p_{*}\right)^{m}$ over $A B, A C$ and $B C$ gives an asymptotic value of $\frac{4}{3} \varphi(m)$, independent of the actual angles $A, B, C$.

In the special case of a vertical triangle the " $A B$ " analysis is as before. For the $A C$ case $p_{*}=(1-y \tan (\alpha / 2))$ is only near 1 if $P$ is near $B$ and the corresponding integral is negligible for large $m$. Also $\left(1-p_{*}\right)^{m} \cong(1-x y / 2)^{m} \cong e^{-m x y / 2}$, and, from (10),

$$
\begin{aligned}
\int\left(1-p_{*}\right)^{m} d P & \cong \iint 2 e^{-m \alpha y / 2}(\alpha-\sin (\alpha)) y(\sin (\alpha))^{-2} d y d \alpha \\
& \cong \iint e^{m \alpha y / 2}(\alpha y / 3) d y d \alpha \cong \frac{4}{3} \varphi(m),
\end{aligned}
$$

again. Consequently, using (1), we have established the following theorem.

Theorem 1. For $n$ lines uniformly and randomly chosen from the convex hull of three non-concurrent lines, with directions confined to a semicircle, then the expected number of extreme lines, $e_{n}$, is asymptotically $2 \ln (n)$ as $n \rightarrow \infty$.

\section{More Than Three Parent Lines}

If the parent polygon is defined by more than three lines the previous analysis will apply at each "corner". Suppose the lines are labelled as follows. Let $g_{1}$ denote the line of minimum orientation (in the range $[0, \pi)$ ) and let $g_{2}, g_{3}, \ldots, g_{s}$ denote the lines of increasing orientation that form the edges of the polygon $R_{0}$ and let $g_{s}, g_{s+1}, \ldots, g_{r}, g_{1}$ be the lines that form the edges of $L_{0}$ with decreasing orientation. A vertex of $R_{0}$ is of the form $g_{i} \cap g_{i+1}(1 \leq i \leq s-1)$; a vertex of $L_{0}$ is of the form $g_{i} \cap g_{i+1}(s \leq i \leq r$, with $r+1$ identified with 1). Two vertices will be adjacent if they are of the form $g_{i} \cap g_{i+1}$ and $g_{i+1} \cap g_{i+2}$ or are $g_{r} \cap g_{1}$ and $g_{1} \cap g_{2}$.

For a point $P$ of $R^{2}-\left(L_{0} \cup R_{0}\right)$ with adjacent support vertices and with $p_{*}$ the probability of a random line of $H_{0}$ having the same orientation about $P$ as $g_{i+1}$ the analysis of the integrals of $p_{*}^{m}$ and $\left(1-p_{*}\right)^{m}$ is the same as in the triangular case, with the former giving negligible contributions and the latter a contribution of $\frac{4}{3} \ln (n)$ to $e_{n}$. For a point with two support vertices which are not adjacent we get negligible contributions. If the two vertices are of the form $g_{i} \cap g_{i+1}$ and $g_{i+2} \cap g_{i+3}$ (indices modulo $r$ ) it is possible to have $p_{*}=0$ but only in the case that the point is $g_{i+1} \cap g_{i+2}$, the argument then is as given in (13) and (14) to conclude that there can only be a negligible contribution. If the two vertices are more than one vertex apart, then $p_{*}$ and $1-p_{*}$ will be both bounded away from 1 (by the measure of an intervening triangle) and so $p_{*}^{m}$ and $\left(1-p_{*}\right)^{m}$ will shrink to zero exponentially fast and be negligible relative to $\varphi(m)$. These considerations - which are essentially the same as in the classic caseallow us to conclude that if the parent polygon $H_{0}$ is generated (non-redundantly) by $r$ 
lines with $r$ vertices to $L_{0} \cup R_{0}$, then, asymptotically, the expected number of extreme lines among $n$ is $\frac{2}{3} r \ln (n)$, that is, the same result as in the classic case.

\section{Discussion of Further Possibilities}

The main technique in this paper dualizes that of Rényi and Sulanke. There is scope for exploring the duals of other classical techniques. As an illustration consider the upper and lower bounds and their asymptotic nature determined by Dwyer (1988) in the simplest case of a 2-gon of lines in the plane; this will consist of the set $\Gamma_{2}$ of lines having the point $A=(1,0)$ on the right and the point $B=(-1,0)$ on the left. Thus $\Gamma_{2}$ consists of lines passing upwards through the interval $[-1,1]$ of the $x$-axis. If we parametrize a line by its orientation $\theta$ and the distance, $p$, that the origin is to the left, then $\Gamma_{2}$ corresponds to the parameter set $\{(p, \theta):-\sin \theta \leq p \leq \sin \theta, 0 \leq \theta \leq \pi\}$, which has total measure 4. We now look for upper and lower bounds for the probability that the first of $n$ random lines in $\Gamma_{2}$ is extreme.

First, any $g_{1}$ can divide $\Gamma_{2}$ into four regions, with $g_{1}$ a vertex of each. We do this by defining two points: the point $X$ is where $g_{1}$ crosses the segment $A B$ and the point $S$ is where $g_{1}$ crosses the vertical line, $s=(-1, \pi / 2)$ through $B$. Let $g_{1}^{\prime}$ denote the line from $S$ to $A$. Then $\Gamma_{2}$ is partitioned into four triangles of lines

$$
\begin{aligned}
\left\langle\left\{e, g_{1}, g_{1}^{\prime}\right\}\right\rangle & =\{g: X \in L(g) ; A, S \in R(g)\}, \\
\left\langle\left\{w, g_{1}, g_{1}^{\prime}\right\}\right\rangle & =\{g: X, S \in L(g) ; A \in R(g)\}, \\
\left\langle\left\{e, g_{1}, s\right\}\right\rangle & =\{g: B \in L(g) ; S, X \in R(g)\}, \\
\left\langle\left\{w, g_{1}, s\right\}\right\rangle & =\{g: B, S \in L(g) ; X \in R(g)\},
\end{aligned}
$$

where $e$ is the line from $A$ to $B$ and $w$ is the line from $B$ to $A$. If $g_{1}$ is in the "first quadrant" ( $p \geq 0, \leq \theta \leq \pi / 2)$, then the first of these has the smallest measure of

$$
S X+A X-A S=\frac{\sin \theta+p}{\sin \theta \cos \theta}+\frac{\sin \theta-p}{\sin \theta}-\sqrt{4+\left(\frac{p+\sin \theta}{\cos \theta}\right)^{2}} \geq t,
$$

where $t$ is defined as $t=\left(\sin ^{2} \theta-p^{2}\right) / 4$ (and so $0 \leq t \leq \frac{1}{4}$ ). If there are $n-1$ other lines in $\Gamma_{2}$, then $g_{1}$ cannot be extreme if all the four triangles above are occupied. Thus for $n$ random lines $G_{1}, G_{2}, \ldots, G_{n}$

$$
\begin{aligned}
\operatorname{Pr}\left(G_{1} \text { is extreme }\right) & \leq \int_{0 \leq \theta \leq \sin \theta, 0 \leq \theta \leq \pi / 2} 4(1-t / 4)^{n-1} d p d \theta \\
& =\int_{-\sin \theta \leq p \leq \sin \theta, 0 \leq \theta \leq \pi / 2} 2(1-t / 4)^{n-1} d p d \theta .
\end{aligned}
$$

In the integral we now change to variables $t$ and $u=(\sin \theta-p) / 2$, so that

$$
d p d \theta=2\left[u^{2}-\left(u^{2}+t\right)^{2}\right]^{-1 / 2} d u d t .
$$


The above inequality becomes

$$
\operatorname{Pr}\left(G_{1} \text { is extreme }\right) \leq 4 \int_{0}^{1 / 4}(1-t / 4)^{n-1} h(t) d t,
$$

where

$$
h(t)=\int_{\alpha_{t}}^{1-\alpha_{t}} 4\left[u^{2}-\left(u^{2}+t\right)^{2}\right]^{-1 / 2} d u,
$$

with $\alpha_{t}=(1-\sqrt{(1-4 t)}) / 2$. In fact $h$ is the probability density function of the statistic $t ; t$ itself seems to play the role of the "product of uniforms" statistic that frequently occurs in classical proofs.

For a lower bound for the probability that $G_{1}$ is extreme we now dualize the technique of associating a hyperplane through a point in a convex region. In our case for a line $g_{1}$ in the first quadrant of $\Gamma_{2}$ we look for a point $X^{*}$ on $g_{1}$ so that the triangle of lines $\left\{g: B, X^{*} \in L(g), A \in R(g)\right\}$ has minimum measure. This triangle of lines has measure $2+A X^{*}-B X^{*}$; this is minimized when

$$
X^{*}=\left(p^{-1} \sin \theta\left(\cos ^{2} \theta+p^{2}\right), p^{-1} \cos \theta\left(\sin ^{2} \theta-p\right),\right.
$$

and the minimum measure becomes simply $2-2 \sqrt{1-4 t}$. Essentially we have determined the "economic cap" (Bárány and Larnman, 1988) through $g_{1}$. If there are $n-1$ other lines in $\Gamma_{2}$, then a sufficient condition for $g_{1}$ to be extreme is that this "cap" is empty. Thus

$$
\operatorname{Pr}\left(G_{1} \text { is extreme }\right) \geq \int_{0}^{1 / 4}\left(\frac{1+\sqrt{1-4 t}}{2}\right)^{n-1} h(t) d t \geq \int_{0}^{1 / 4}(1-2 t)^{n-1} h(t) d t .
$$

Consequently we can argue that the expected number, $e_{n}$, of extreme lines amongst $n$ in $\Gamma_{2}$ is bounded by $L_{n} \leq e_{n} \leq U_{n}$, where

$$
L_{n}=n \int_{0}^{1 / 4}(1-2 t)^{n-1} h(t) d t \quad \text { and } \quad U_{n}=4 n \int_{0}^{1 / 4}(1-t / 4)^{n-1} h(t) d t .
$$

To provide simple estimates of $h(t)$, write $Q_{1}=u-u^{2}-t$ and $Q_{2}=u+u^{2}+t$, so that $Q_{1} Q_{2}$ is the quartic in the denominator of the $h(t)$ integral. For $\alpha_{t} \leq u \leq 1$,

$$
Q_{1} \leq u-\alpha_{t}, \quad Q_{2} \leq 2 u+t
$$

thus

$$
\begin{aligned}
h(t) & \geq \int_{\alpha_{t}}^{1 / 2} 4\left[\left(u-\alpha_{t}\right)(2 u+t)\right]^{-1 / 2} d u \\
& =2 \sqrt{2}\left\{\ln \left(2 \sqrt{2\left(1-2 \alpha_{t}\right)(1+t)}+2+t-2 \alpha_{t}\right)-\ln \left(t+2 \alpha_{t}\right)\right\} \geq 2 \sqrt{2} \ln (1 / 4 t) .
\end{aligned}
$$

Also

$$
Q_{1} \geq \frac{1-4 t}{2\left(1-2 \alpha_{t}\right)} \min \left\{\left(u-\alpha_{t}\right),\left(1-\alpha_{t}-u\right)\right\}, \quad Q_{2} \geq u+t,
$$




$$
\begin{aligned}
\int_{\alpha_{t}}^{1-\alpha_{t}} \frac{1}{\sqrt{Q_{1} Q_{2}}} d u \leq & \sqrt{\frac{2\left(1-2 \alpha_{t}\right)}{1-4 t}} \\
& \times\left(\int_{\alpha_{t}}^{1 / 2} \frac{1}{\sqrt{\left(u-\alpha_{t}\right)(u+t)}} d u+\int_{1 / 2}^{1-\alpha_{t}} \frac{1}{\sqrt{\left(1-u-\alpha_{t}\right)}} d u\right) \\
\leq & 2 \sqrt{\frac{2\left(1-2 \alpha_{t}\right)}{1-4 t}} \int_{\alpha_{t}}^{1 / 2} \frac{1}{\sqrt{\left(u-\alpha_{t}\right)(u+t)}} d u \\
= & 2 \sqrt{\frac{2\left(1-2 \alpha_{t}\right)}{1-4 t}}\left\{\ln \left[\sqrt{\left(1-2 \alpha_{t}\right)(1+2 t)}+1+t-\alpha_{t}\right]\right. \\
& \left.-\ln \left[\alpha_{t}+t\right]\right\} .
\end{aligned}
$$

The last expression can be shown to be no greater than $2 \sqrt{10} \ln (9 / 8 t)$ and so a fairly inefficient upper bound for the $h(t)$ is $8 \sqrt{10} \ln (9 / 8 t)$.

Thus

$$
L(n) \geq n \int_{0}^{1 / 4} 2 \sqrt{2} \ln (1 / 4 t)(1-2 t)^{n-1} d t \approx \sqrt{2} \ln (n),
$$

the last approximation is "standard" and follows the same lines as in the classical case (see, for example, Dwyer, 1988). Also

$$
U(n) \leq 32 \sqrt{10} n \int_{0}^{1 / 4}(1-t / 4)^{n-1} \ln (9 / 8 t) d t \approx 128 \sqrt{10} \ln (n)
$$

It follows, by the arguments of this section, that $e_{n}=\Omega(\ln (n))$, as in the classical case (here only for the 2-gon).

Other classical methods may be adaptable; the approach of Groeneboom uses the boundary process indexed by support lines of a point set. The dual approach would use a boundary process of lines indexed by the points of $\partial\left(L_{0} \cup R_{0}\right)$.

A Poisson process line is familiar, but re-scaling of sets of lines may present a problem as may the (probably) more complex expressions for Poisson void probabilities, which Groeneboom found amenable.

The ideas here extend to hyperplanes in higher dimensions. In Gates (to appear) the three- and higher-dimensional Sylvester-Klee problem is discussed with some numerical, but no asymptotic results.

Finally we note that the definition of convex hulls of (directed) lines used here is different to that introduced by Goodman and Pollack (1995); with their definition a general triad of lines is already convex (and so the expected measure of its hull is zero). 


\section{Acknowledgment}

I thank the referee for corrections and helpful suggestions.

\section{References}

F. Affentranger and J. A. Wieacker (1991), On the convex hull of uniform random points in a simple $d$-polytope, Discrete Comput. Geom. 6, 291-305.

I. Bárány and C. Buchta (1993), Random polytopes in a convex polytope, independence of shape, and concentration of vertices, Math. Ann. 297, 467-497.

I. Bárány and D. G. Larman (1988), Convex bodies, economic cap coverings, random polytopes, Mathematika 35, 274-291.

C. Bauer and R. Schneider (1995), Extremal problems for geometric probabilities involving convex bodies, Adv. in Appl. Probab. 27, 20-34.

C. Buchta (1985), Zufällige Polyeder-Eine Übersicht, In Zahlentheoretische Analysis, ed. E. Hlawka, Lecture Notes in Mathematics, Vol. 1114, Springer-Verlag, Berlin, pp. 1-13.

C. Buchta and M. Reitzner (1992), What is the expected volume of a tetrehedron whose vertices are chosen at random from a given tetrahedron? Anz. Österreich. Akad. Wiss. Math.-Natur. Kl. 129, 63-68.

C. Buchta and M. Reitzner (1997a), Equiaffine inner parallel curves of a plane convex body and the convex hulls of randomly chosen points, Probab. Theory Relat. Fields 108, 385-415.

C. Buchta and M. Reitzner (1997b), On a theorem of G. Herglotz, Rend. Circ. Mat. Palermo (2) Suppl. 50, 89-102.

A. J. Cabo and P. Groeneboom (1994), Limit theorems for functionals of convex hulls, Probab. Theory Relat. Fields 100, 31-55.

R. A. Dwyer (1988), On the convex hull of random points in a polytope, J. Appl. Probab. 25, 688-699.

J. Gates (1993), Some dual problems of geometric probability in the plane, Combin. Probab. Comput. 2, 11-23.

J. Gates (to appear), Dual convexity in space and the Sylvester-Klee problem, Far Eastern J. Math. Sci.

A. A. Giannopoulos (1992), On the mean value of the area of a random polygon in a plane convex body, Mathematika 39, 279-290.

J. E. Goodman and R. Pollack (1995), Foundations of a theory on convexity on affine Grassmann manifolds, Mathematika 42, 305-328.

P. Groeneboom (1988), Limit theorems for convex hulls, Probab. Theory Relat. Fields 79, 327-368.

D. Mannion (1994), The volume of a tetrahedron whose vertices are chosen at random in the interior of a parent tetrahedron, Adv. in Appl. Probab. 26, 577-596.

A. Rényi and R. Sulanke (1963), Über die konvexe Hülle von $n$ zufällig gewählten punkten, Z. Wahrsch. Verw. Gebiete 2, 75-84.

L. A. Santalaló (1976), Integral Geometry and Geometric Probability, Encyclopedia of Mathematics and its Applications, Vol. 1, Addison-Wesley, Reading, MA.

R. Schneider (1988), Random approximation of convex sets, J. Microscopy 151, 211-227.

R. Schneider (1997), Discrete aspects of stochastic geometry, in Handbook of Discrete and Convex Geometry, CRC Press, New York.

W. Weil and J. A. Wieacker (1993), Stochastic geometry, in Handbook of Convex Geometry, eds. P. M. Gruber and J. M. Wills, Elsevier, Amsterdam, pp. 1391-1438.

R. Wong (1989), Asymptotic Approximation of Integrals, Academic Press, London.

Received June 29, 2000, and in revised form December 21, 2001. Online publication March 27, 2002. 